\section{AB0058 LEVEL OF VASCULAR ENDOTHELIAL GROWTH FACTOR A AND INTIMA-MEDIA THICKNESS IN PATIENTS WITH RHEUMATOID ARTHRITIS AND OSTEOARTHRITIS}

V.O. Omelchenko, E.A. Letyagina, M.A. Korolev. FSBSI "Scientific Institute of clinical and experimental lymphology", Novosibirsk, Russian Federation

Background: Early development of atherosclerosis (AS) with higher mortality risk is observed in patients with rheumatoid arthritis (RA). Actually, this problem hasn't been solved. That's why researches devoted to finding of relationships between RA and AS is still of current interest. In RA pannus formation accompanied by the development of new blood vessels forms main clinical manifestation. Vascular endothelial growth factor A (VEGF-A) is a heparin-binding glycoprotein, that induces the growth of blood vessels and plays a role in differentiation of endothelial cells. The expression of VEGF-A increases when plaque developes. Objectives: To determine the particular qualities of VEGF-A production in patients with RA and various atherosclerotic damage of vessels.

Methods: 67 Caucasian patients with RA (age - 52 yr., [38;59], DAS28 $5,25[5,5 ; 6,4])$ were included in our study. Patients had American College of Rheumatology (ACR)-defined RA (1987 classification criteria). 45 Europeoid patients with OA (56 yr., [50;63]) were in control group. All patients gave written informed consent before enrollment. Level of VEGF-A was determined by ELISA for quantitative detection of human VEGF-A (Bender MedSystem GmbH, Austria). Range of atherosclerotic damage was assessed by ultrasonography with measurement of carotid intima-media thickness (IMT). IMT measured was compared with normal values according to the age and sex classes: younger $40 \mathrm{yr}-0,7 \mathrm{~mm}, 40-50 \mathrm{yr}-0,8 \mathrm{~mm}$, elder $50 \mathrm{yr}-0,9 \mathrm{~mm}$ for men; younger $45 \mathrm{yr}-0,7 \mathrm{~mm}, 45-60 \mathrm{yr}-0,8 \mathrm{~mm}$, elder $60 \mathrm{yr}-0,9 \mathrm{~mm}$ for women. Results is presented as median and 25 th/75th percentiles (Me [25th percentile; 75 th percentile]. Descriptive statistics, non-parametric Mann-Whitney U-test, Kruskal Wallis test were used for analysis of the results.

Results: No significant differences of IMT were found between groups with RA and OA. Abnormal IMT were observed in about half of patients (Table 1).

Table 1. IMT in patients with RA and OA

\begin{tabular}{lccc}
\hline & IMT & IMT $>$ N & Plaque \\
\hline RA, $n(\%)$ & $35(52.2)$ & $20(29.9)$ & $12(17.9)$ \\
OA, $n(\%)$ & $18(40)$ & $12(26.7)$ & $15(33.3)$ \\
\hline
\end{tabular}

The highest level of VEGF-A was in OA-group with stenosing plaque $(p<0,01)$. No significant differences were found between other OA-subgroup. In patients with RA the highest level of VEGF-A was observed in subgroup with abnormal IMT $(p<0,05)$, but not with pluques. Generally, VEGF-A production in patients with normal IMT was higher in RA-patients, but not sugnificially.

Table 2. Level of VEGF-A in groups with different values of IMT

\begin{tabular}{ccccc}
\hline & IMT & IMT $>$ N & $\begin{array}{c}\text { Plaque without } \\
\text { stesosis }\end{array}$ & $\begin{array}{c}\text { Stenosing } \\
\text { plaque }\end{array}$ \\
\hline $\begin{array}{c}\text { VEGF-A in OA-patients, } \\
\text { pg/ml }\end{array}$ & 392,30 & 396,90 & 651,05 & 1148,50 \\
VEGF-A in RA-patients, & {$[225,60 ; 641,33]$} & {$[213,08 ; 519,95]$} & {$[318,58 ; 1041,25]$} & {$[1041,3 ; 1164,25]$} \\
pg/ml & 753,6 & 1013 & 837,25 & 712,75 \\
\hline
\end{tabular}

Conclusions: Regulation of VEGF-A production may play a role in earlier onset of atherosclerosis in patients with RA.

Disclosure of Interest: None declared

DOI: 10.1136/annrheumdis-2017-eular.4556

\section{Cartilage, synovium and osteoimmunology}

\section{AB0059 SYNOVIAL SECRETION OF PRO-INFLAMMATORY AND PRO-OXIDANT MOLECULES TRIGGERED BY MONOSODIUM URATE CRYSTALS INDUCES NGF AND H2O2 PAIN MEDIATORS IN THE CHONDROCYTE}

A.G. Lopez-Reyes ${ }^{1}$, D. Medina-Luna ${ }^{2}$, M. Satamaria-Olmedo ${ }^{2}$,

K. Martinez-Flores ${ }^{3}$, Y. Zamudio-Cuevas ${ }^{3}$, J. Fernandez-Torres ${ }^{3}$,

G. Martinez-Nava ${ }^{3}$, D. Clavijo-Cornejo ${ }^{3}$, A. Olivos-Meza ${ }^{4}$, M. Gutierrez $^{5}$

M. Fernandez-Moreno ${ }^{6}$, F. Blanco ${ }^{6}$, C. Pineda ${ }^{5} .{ }^{1}$ Laboratorio de Liquido

Sinovial, Instituto Nacional de Rehabilitación "LGII"; ${ }^{2}$ Laboratorio de Liquido

Sinovial, Instituto Nacional de Rehabilitacion "LGII"; ${ }^{3}$ Laboratorio de Liquido Sinovial, Instituto Nacional de Rehabilitacion "LGII"; ${ }^{4}$ Arthroscopy Service, Instituto Nacional de Rehabilitación "LGII"; ${ }^{5}$ Department of Rheumatic and Musculoskeletal Diseases, Instituto Nacional de Rehabilitacion "LGII", Mexico City, Mexico; ${ }^{6}$ Rheumatology Service, INIBIC, CIBER-BBN, A Coruña, Spain

Background: Gout is the most prevalent inflammatory arthropathy in young men, in which clinical manifestations are triggered by the deposits of monosodium urate (MSU) crystals in and around joints. From a clinical perspective, gouty attacks and tophaceous deposits have been associated to nodal osteoarthritis $[1,2]$.

Objectives: To determine the molecular mechanisms associating MSU crystal deposits with chondrocyte activation and changes in articular cartilage.

Methods: Primary cultures of synoviocytes were activated with MSU crystals for $24 \mathrm{~h}$, the resulting supernatant was used as a supplementary conditioning basal medium (CBM) for $24 \mathrm{~h}$ chondrocyte growth. The expression level profile of cytokines (IL-6, IL-8) and nerve growth factor (NGF) in the supernatant was measured. Production of reactive oxygen and nitrogen species, such as $\mathrm{O}_{2}^{\mathbf{*}}$, $\mathrm{H}_{2} \mathrm{O}_{2}$, and $\mathrm{NO}^{*}$, was also measured intracellularly.

Results: Upon activation by MSU crystals, synoviocytes increased secretion of IL-6 and IL-8, as well as intracellular production of $\mathrm{O}_{2}^{\bullet}, \mathrm{H}_{2} \mathrm{O}_{2}$, and $\mathrm{NO}^{\bullet}$ in comparison to non-stimulated synoviocytes. Additionally, the chondrocytes incubated with CBM for $24 \mathrm{~h}$ increased significantly IL-6, IL-8, and NGF levels, as well as $\mathrm{O}_{2}^{\bullet}, \mathrm{H}_{2} \mathrm{O}_{2}$, and $\mathrm{NO}^{\bullet}$ levels when compared to non-stimulated chondrocytes. Secretion of these soluble mediators was even higher than the one observed in the synoviocytes and chondrocytes directly stimulated with MSU crystals.

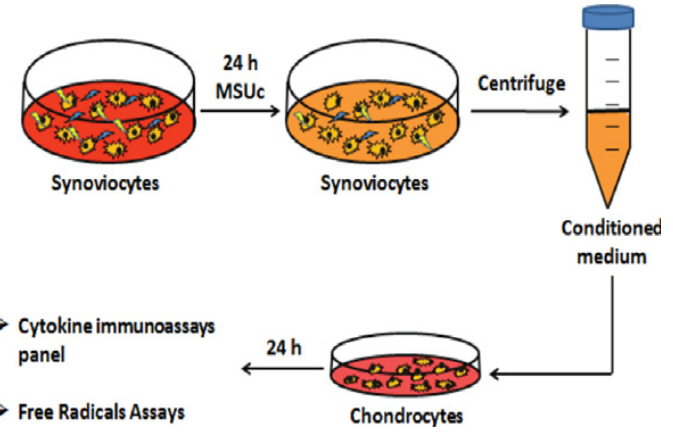

Conclusions: Pro-inflammatory cytokine secretion induced by MSU crystals in synoviocytes triggers chondrocyte activation, intensifying the articular inflammatory state in gouty attacks. Furthermore, the increase in reactive species (particularly $\mathrm{NO}^{\bullet}$, which inhibits proteoglycan synthesis) compromises the homeostasis of the extracellular matrix of the cartilage. Finally, overproduction of NGF and $\mathrm{H}_{2} \mathrm{O}_{2}$ by the chondrocyte functions a pain modulator during gout attacks. References:

[1] Fam AG, et al. Gouty arthritis in nodal osteoarthritis. J Rheumatol 1996; 23:684-9.

[2] Roddy E, et al. Gout and nodal osteoarthritis: a case-control study. Rheumatology (Oxford) 2008; 47:732-3.

Disclosure of Interest: None declared

DOI: 10.1136/annrheumdis-2017-eular.2401

\section{AB0060 MODELLING DELAYED BONE HEALING IN A MOUSE- OSTEOTOMY MODEL TO EVALUATE THERAPEUTIC STRATEGIES FOR AFFECTED PATIENTS WITH RA}

A. Lang ${ }^{1,2,3}$, S. Fügener ${ }^{1}$, P. Hoff ${ }^{1}$, K. Schmidt-Bleek ${ }^{4}$, T. Gaber $^{1,3}$ F. Buttgereit ${ }^{1,3} .{ }^{1}$ Department of Rheumatology and Clinical Immunology; ${ }^{2}$ Berlin-Brandenburg School for Regenerative Medicine, Charité University Hospital; ${ }^{3}$ German Arthritis Research Center; ${ }^{4}$ Julius Wolff Institute and Center for Musculoskeletal Surgery, Charité University Hospital, Berlin, Germany

Background: Anti-inflammatory treatment of rheumatoid arthritis (RA) using non-steroidal anti-inflammatory drugs (NSAID) or glucocorticoids (GC) as well as the disease itself, are supposed to negatively influence bone metabolism and healing. However, in vivo models allowing to evaluate therapeutic strategies for patients suffering from delayed bone healing are scarce and mainly created by critical size defects that are not representative for comorbity-induced disorders. In addition, there are no adequate rodent models allowing the analyses of the influence on the bone metabolism by the complete dysregulation of the immune system in RA as well as long-term medications with NSAID or GC. Previously, we have shown that immunologically restricted patients lack an adequate adaptation to hypoxia in the fracture hematoma thus facilitating healing disorders (1). The hypoxic microenvironment during the initial fracture-healing phase is known to be essential for activating the immunological reactions, which induce the regeneration. Furthermore, we performed a single-center retrospective study on fracture healing disorders showing a significant high prevalence for RA patients to be affected by delayed bone healing.

Objectives: In order to evaluate therapeutic strategies for affected patients with RA, we developed a model for delayed bone healing in a mouse-osteotomy model.

Methods: Female C57BL aged 12 weeks underwent osteotomy of the femur (fracture gap $0,7 \mathrm{~mm}$ ) that was fixated with an external fixator (RIS-System). Lyostypt $^{\circledR}$ (based on bovine Col I; mimicking extracellular bone matrix) was applied in the fracture gap and analysis was performed 2 and 3 weeks after surgery. The ratio of bone volume (BV) per total volume (TV) in the fracture gap was evaluated using in vitro $\mu \mathrm{CT}$. In addition, Movat's pentachrome staining was performed to analyze the cellular and tissue composition within the fracture gap. To investigate the number of cells as well as the vessel formation, we used immunofluorescence to stain for Endomucin, PECAM-1 and DAPI. Quantitative analysis of histological staining was conducted by using ImageJ.

Results: We developed an in vivo mouse-osteotomy model showing delayed bone healing by applying Lyostypt ${ }^{\circledR}$ in the fracture gap. The BV, TV as well as the 